\title{
Genome-wide analysis of androgen receptor binding sites in prostate cancer cells
}

\author{
YUE CHENG, PAN YU, XIUZHI DUAN, CHUNHUA LIU, SIQI XU, YUHUA CHEN, \\ YUNNIAN TAN, YUN QIANG, JUNFANG SHEN and ZHIHUA TAO \\ Department of Laboratory Medicine, The Second Affiliated Hospital, \\ Zhejiang University School of Medicine, Hangzhou, Zhejiang 310009, P.R. China
}

Received July 20, 2014; Accepted March 20, 2015

DOI: $10.3892 /$ etm.2015.2406

\begin{abstract}
The transformation of prostate cancer from an androgen-dependent state to an androgen-independent state is a lethal progression. Alterations in transcriptional programs are the basis of prostate cancer deterioration. The androgen receptor (AR), a member of the nuclear hormone receptor superfamily, mediates prostate cancer progression by functioning primarily through the ligand-activated transcription of target genes. Therefore, a detailed map of AR-regulated genes and AR genomic binding sites is required for hormone-naive and castration-resistant prostate cancer. Through the use of chromatin immunoprecipitation in combination with direct sequencing, 4,143 AR binding sites were defined in the LNCaP androgen-sensitive prostate cancer cell line. Using the same method, 2,380 AR binding regions were identified in the LNCaP-AI long-term androgen-deprived cell line. Approximately $8.5 \%(354 / 4,143)$ of the binding regions were mapped to within $2 \mathrm{~kb}$ of the transcription start site (TSS) in the LNCaP cells, while $\sim 12.6 \%(299 / 2,380)$ were mapped to within $2 \mathrm{~kb}$ of the TSS in the LNCaP-AI cells. In total, the study mapped 2,796 genes in LNCaP cells and 1,854 genes in LNCaP-AI cells. The cell lines shared 789 mutual genes. In addition, gene ontology (GO) analysis of the genes revealed that there was a notable overlap between the GO terms in the LNCaP cells and LNCaP-AI cells. However, GO terms within the biological process domain that were only observed in the LNCaP-AI cells included the reproduction process, death, immune system process, multi-organism process, pigmentation and viral reproduction. The major genes in the different GO terms were TNFAIP8, RTN4, APP and SYNE1. Through
\end{abstract}

Correspondence to: Professor Zhihua Tao, Department of Laboratory Medicine, The Second Affiliated Hospital, Zhejiang University School of Medicine, 88 Jiefang Road, Hangzhou, Zhejiang 310009, P.R. China

E-mail: zrtzh@zju.edu.cn

Key words: androgen receptor, chromatin immunoprecipitation, high-throughput sequencing, androgen receptor binding sites, prostate cancer analyzing the AR binding sites in the two cell types, the present study aimed to map potential AR-regulated genes, identify their associated transcription factors and provide a new perspective on the biological processes in the development of prostate cancer. The results provided a valuable data set that furthered the understanding of the genome-wide analysis of AR binding sites in prostate cancer cells, which may be exploited for the development of novel prostate cancer therapeutic strategies.

\section{Introduction}

The androgen receptor (AR) is a member of the nuclear receptor superfamily that functions as a ligand-dependent transcription factor and plays an important biological role in the male phenotype and prostate cancer biology. Prostate cancer was originally identified as an androgen-dependent tumor, whose growth and survival were under the control of AR signaling (1). Androgen deprivation therapy is initially effective for inhibiting the growth of prostate cancers by suppressing AR activity (2); however, the possibility of recurrence is relatively high and the recurrence is associated with androgen independence (3). Despite a loss of response to antiandrogens, there are data indicating that AR signaling continues to function in castration-resistant prostate cancer (CRPC). The mechanisms include: i) AR following an adaptive process by an activating mutation or multiplication in response to androgen deficiency (4-6); ii) AR regulating other signaling pathways to execute the procedure of self-activation (7); and iii) transcription activities of AR activated by AR signals. Additionally, alternative signaling pathways may result in the activation of the AR $(8,9)$. These observations support a strong selective pressure to maintain AR-regulated signaling pathways in castration-resistant forms of the disease.

Previous studies have aimed to identify androgen-regulated genes or AR genomic binding sites (10-12). In the process where androgen-dependent prostate cancer (ADPC) turns into androgen-independent prostate cancer (AIPC), the effect of an activated AR and associated gene differential expression remains unclear. The AR may be found in different binding molecules within the nucleus in the development of prostate cancer (1); therefore, a detailed 
map of AR-regulated genes and AR genomic binding sites in the hormone-naive and CRPC forms of prostate cancer is required. To determine the changes between AR-dependent and AR-independent chromatin accessibility, chromatin immunoprecipitation in combination with direct sequencing (ChIP-seq) was performed on the well-established LNCaP androgen-sensitive prostate cancer cell line and the long-term LNCaP-AI androgen-deprived cell line, following hormone induction. A high-throughput elucidation of these sites allowed for a deep understanding of the complexities of this process. In the present study, an interdisciplinary approach was taken to successfully discern direct gene targets of the AR. The results indicated that AR genomic binding sites in LNCaP-AI cells exposed to $10 \mathrm{nM}$ dihydrotestosterone differ compared with those in LNCaP cells. The aim of the study was to provide an essential reference for the development of novel biomarkers and potential diagnostic biomarkers to improve the current methods of therapeutic intervention and treatment.

\section{Materials and methods}

Cell culture. The prostate cancer cell line, $\mathrm{LNCaP}$, was obtained from the American Type Culture Collection (Manassas, VA, USA) and routinely cultured in Dulbecco's modified Eagle's medium (DMEM) with $10 \%$ fetal bovine serum (FBS; Gibco Life Technologies, Carlsbad, CA, USA) at $37^{\circ} \mathrm{C}$ in $5 \% \mathrm{CO}_{2}$. The establishment of an androgen-independent cell line (LNCaP-AI) was conducted as previously described (13). The LNCaP-AI cells were grown under phenol red-free DMEM with $10 \%$ dextran-charcoal stripped FBS (dcc-FBS; Gibco Life Technologies) (14).

ChIP assay. ChIP assays were performed using an EZ-Zyme ${ }^{\mathrm{TM}}$ Chromatin Prep kit (17-375; Millipore Corporation, Billerica, MA, USA) and a Magna ChIP ${ }^{\mathrm{TM}}$ A-Chromatin Immunoprecipitation kit (17-610; Millipore Corporation). The cells were cultured in dcc-FBS medium without phenol red for 3 days prior to stimulation with $10 \mathrm{nM}$ dihydrotestosterone (Sigma-Aldrich, St. Louis, MO, USA) for $24 \mathrm{~h}$. Approximately $4 \times 10^{7}$ cells were used for the ChIP assay. Chromatin was prepared from the LNCaP and LNCaP-AI cells $\left(\sim 4 \times 10^{7}\right)$, according to the manufacturer's instructions. The cells were fixed with $550 \mu 1$ formaldehyde (1\%) for $10 \mathrm{~min}$ at room temperature, and glycine $(2 \mathrm{ml})$ was added to quench the unreacted formaldehyde. The fixed cells were resuspended in $450 \mu \mathrm{l}$ EZ-Zyme lysis buffer containing $5 \mu \mathrm{l}$ protease inhibitor cocktail. Subsequently, the nuclei were collected by centrifugation at $2,500 \mathrm{x} \mathrm{g}$, and resuspended in $150 \mu \mathrm{l}$ digestion buffer (\#17-375; Millipore Corporation). Samples were sheared to an average DNA length of 100-500 bp using $10 \mu$ l EZ-Zyme enzymatic cocktail. The lysates were rotated at $4^{\circ} \mathrm{C}$ overnight with $10 \mu \mathrm{l}$ rabbit polyclonal AR antibody (\#17-10489; Millipore Corporation). Protein G beads (50 $\mu$ 1; \#88848; Life Technologies, Carlsbad, CA, USA) were added and incubated for $2 \mathrm{~h}$, after which the precipitates were eluted from the beads with $100 \mu \mathrm{l}$ ChIP elution buffer. Cross-links were reverted by heating at $62^{\circ} \mathrm{C}$ overnight, and DNA was recovered using a QIAquick Polymerase Chain Reaction Purification kit (Qiagen GmbH, Hilden, Germany).
ChIP-seq data analysis. ChIP DNA fragments were processed for deep sequencing at a 49-bp read length on the Illumina Genome Analyzer System (Illumina HiSeq 2000 platform; Illumina, Chesterford, UK). Sequence tags were obtained and mapped to the human genome using the Solexa Analysis Pipeline (Illumina). The output of the Solexa Analysis Pipeline was converted to browser extensible data files for viewing the data in the University of California at Santa Cruz (UCSC) genome browser. Model-based analysis for ChIP-seq (MACS) (15) was used with default parameters to detect the statistically significant peaks of mapped reads. The results were mapped to human genome version 19 (hg19), and the peaks were denoted as high-confidence AR binding sites (ARBSs). The high-confidence ARBSs were each split into five categories according to the peak locations, namely an intragenic region, exons, introns, upstream $20 \mathrm{~kb}$ and downstream $20 \mathrm{~K}$. To calculate the distance to the transcription start sites (TSS), annotations from the UCSC genome browser were used. The data were available from the National Center for Biotechnology Information Gene Expression Omnibus under the accession number GSE44800. Using a stringent P-value cutoff of $0.00001,2,800 \mathrm{AR}$ ChIP-enriched regions were identified as significant ARBSs.

Motif scanning and identification. To determine the motif enrichment in regions of AR bound sequences, Multiple EM for Motif Elicitation (MEME; version 4.6.1) transcription factor binding site motif identification software was used (16). MEME is a web-based tool for analyzing motifs in large DNA data sets. The software performs de novo motif discovery, motif enrichment analysis and motif location analysis, providing a comprehensive image of the DNA motif in the LNCaP and LNCaP-AI sequence tags (17). The matching criterion was set at a likelihood ratio (LR) of $\geq 500$, and matched matrices with a LR of $\geq 500$ were tabulated. MEME also compared each of the matched matrices with each of the motifs in the JASPAR CORE database.

Gene ontology analysis. The Blast2GO suites were used to determine gene ontology (GO) function categories, while GO enrichment analysis was performed using Web Gene Ontology Annotation Plot (WEGO) software (18). The observed number of differentially expressed transcripts in each GO category was compared with the corresponding number in the UniGene database (http://www.ncbi.nlm.nih.gov/unigene/) to assess the significant over-representation of differentially expressed transcripts in the GO categories. Statistical significance of over-representation for each GO category was determined using Pearson's $\chi^{2}$ test, where $\mathrm{P}<0.05$ was considered to indicate a statistically significant difference. For LNCaP and LNCaP-AI samples, the genes bound by the AR in an exon or intron region of the gene or, at most, $50 \mathrm{~K}$ upstream of the TSS or $50 \mathrm{~K}$ downstream of the 3 -untranslated region, were collected into separate lists. The gene lists were subsequently used to identify statistically over-represented GO terms with WEGO. GO has three domains, namely molecular function, cellular component and biological process. The basic unit of $\mathrm{GO}$ is a term, and each GO term belongs to one of the three domains. 


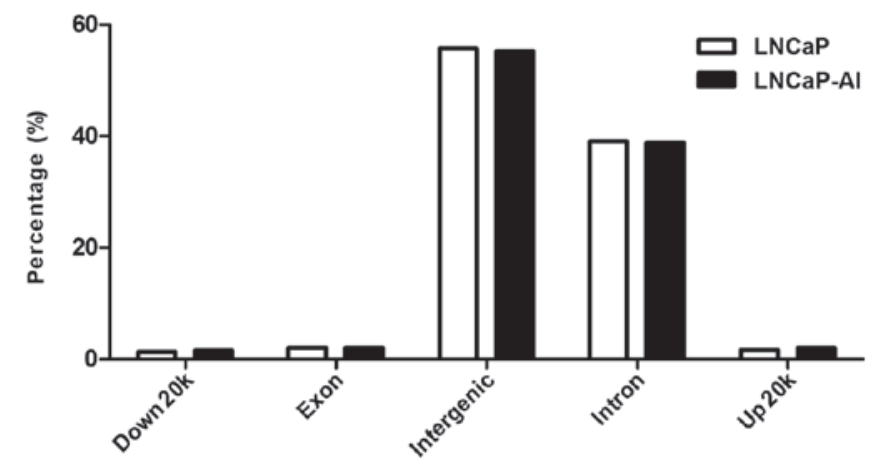

Figure 1. Peak distribution in the gene elements. Peaks were classified based on the location (University of California at Santa Cruz annotation data) and were divided into the following genome regions: Intergenic, intron, downstream, upstream and exon.

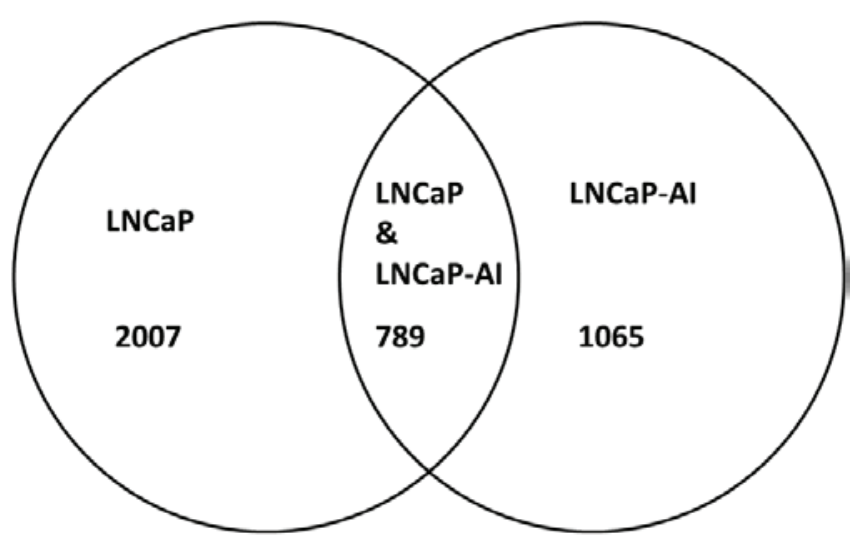

Figure 2. Genetic intersection of LNCaP and LNCaP-AI cells. The Venn diagram shows that $\mathrm{LNCaP}$ and $\mathrm{LNCaP}-\mathrm{AI}$ cells have 2,796 and 1,854 genes, respectively, in which 789 genes form the genetic intersection set of the two cell lines.

\section{Results}

Global identification of direct AR-regulated genes. In order to identify direct AR-regulated genes in the two cell lines that represented distinct molecular subtypes of prostate cancer, the ligand-dependent (LNCaP) and ligand-independent (LNCaP-AI) subtypes, ChIP-seq of the DNA from anti-AR ChIP was performed. To improve the sensitivity of the approach, the AR ChIP-seq in the two cell lines were performed in the presence of dihydrotestosterone in the two cell lines. Using ChIP-seq, 31,115,845 sequence tags in the LNCaP cells and 9,414,950 sequence tags in the LNCaP-AI cells were obtained, which were mapped uniquely to hg19 allowing two mismatches. By generating peak range scanning with the use of MACS software, 9,021 and 4,949 peaks were obtained in the LNCaP and LNCaP-AI cells, respectively, which were confirmed with the Poisson Distribution Model $(\mathrm{P}<0.00001)$. The distribution character of these peaks is presented in Fig. 1. Using model-based analysis for ChIP-seq, 4,143 ARBSs were identified in LNCaP cells and 2,380 AR binding regions were identified in LNCaP-AI cells, based on a stringent false discovery rate (FDR) of 0.05 . LNCaP cells were found to have a greater number of higher affinity ARBSs compared with LNCaP-AI cells, which is consistent with a previous study
Table I. List of genes according to GO biological processes.

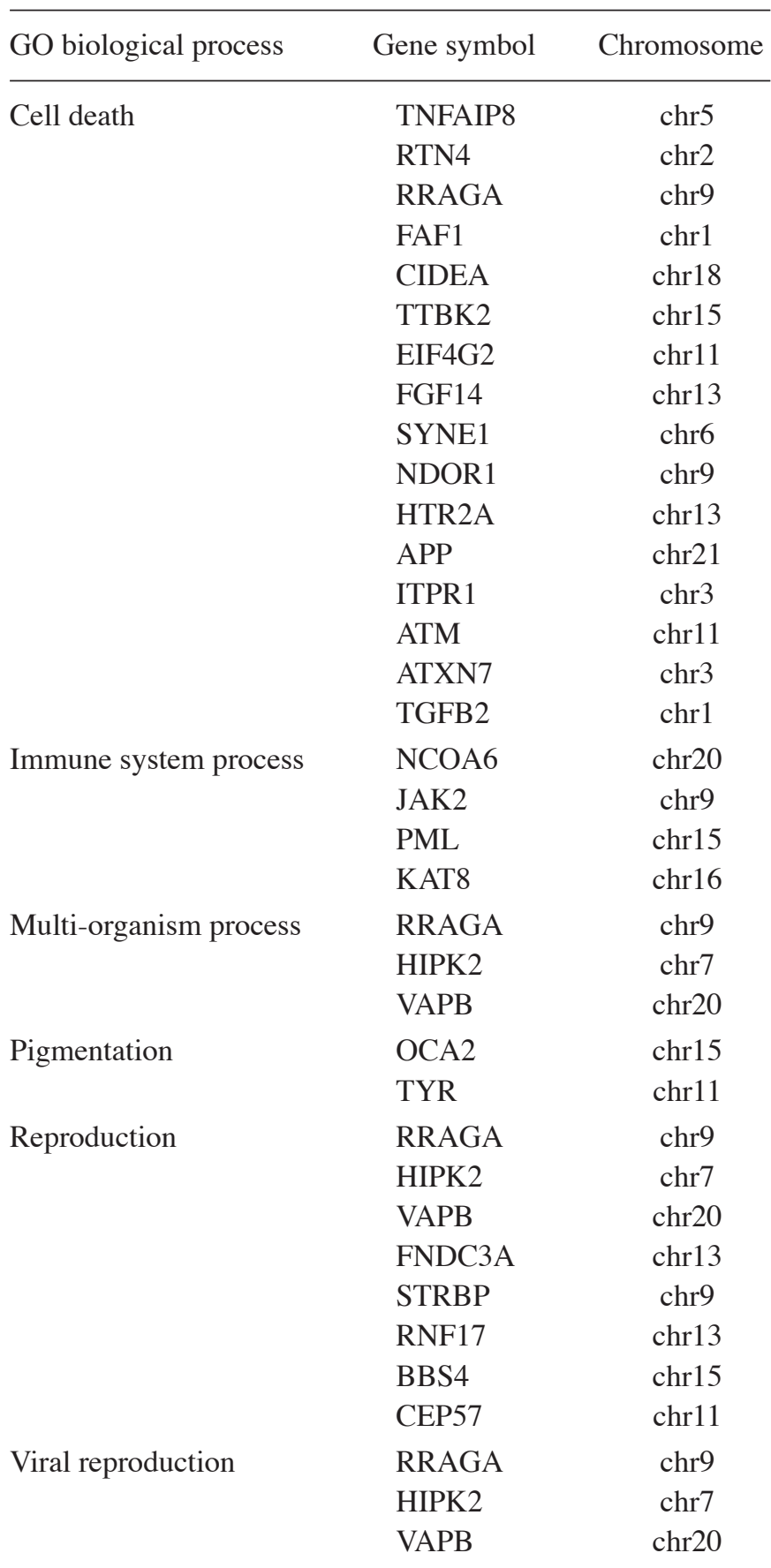

GO, gene ontology.

in which androgen signaling activity was demonstrated to be decreased in AIPC compared with ADPC (19). As shown in Fig. 2, 4,143 AR binding regions in the LNCaP cells and 2,261 AR binding regions in the LNCaP-AI cells were mapped to their corresponding TSSs of 2,796 genes and 1,854 genes, respectively. Furthermore, the two cell lines (LNCaP and LNCaP-AI) were shown to share 789 mutual genes, and the average numbers of AR binding regions per gene were determined as 1.49 for $\mathrm{LNCaP}$ and 1.28 for LNCaP-AI.

Functional characterization of genes in the LNCaP and LNCAP-AI cells. To elucidate the functions of the 


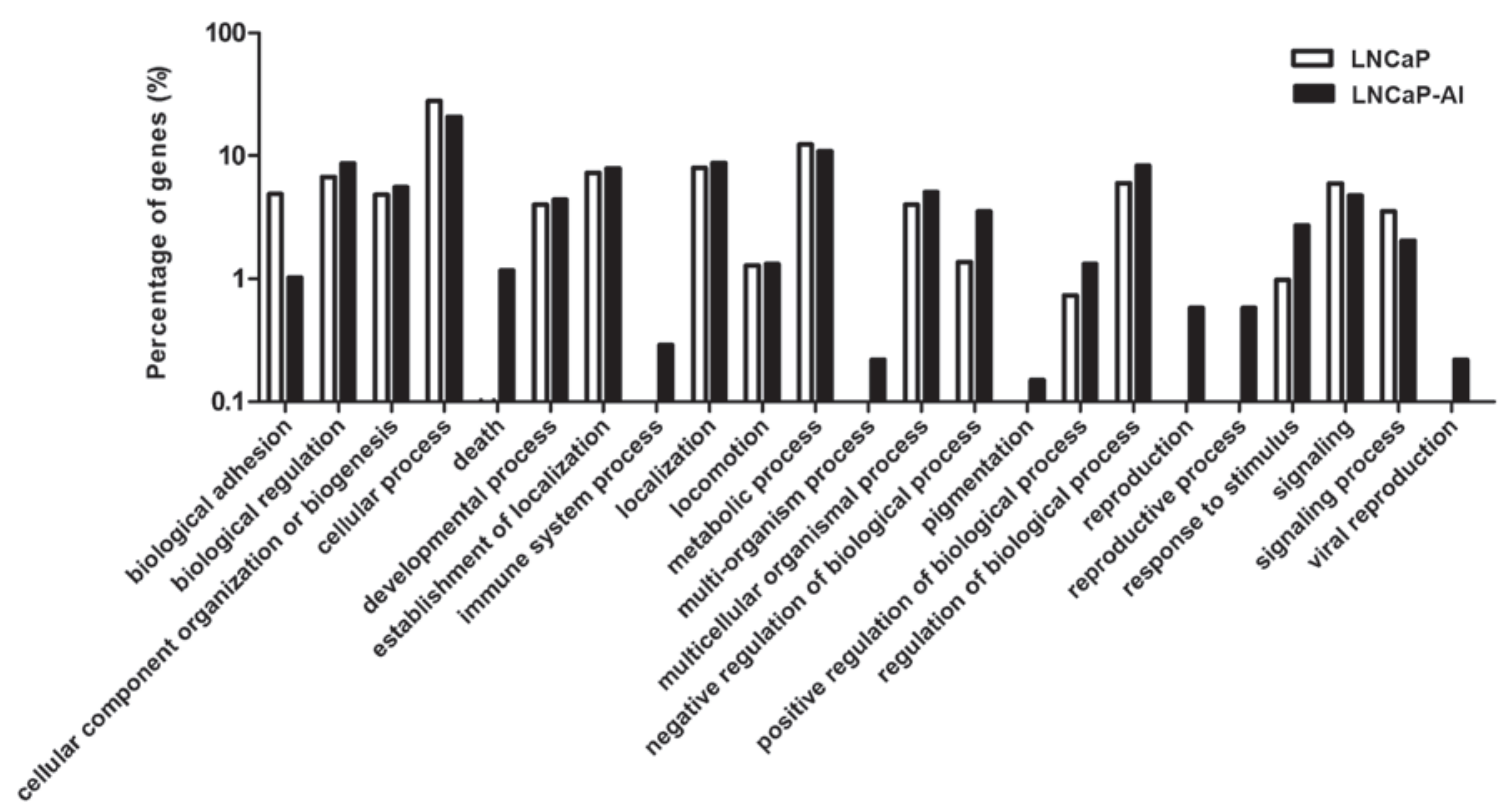

Figure 3. Functional annotation of androgen receptor chromatin immunoprecipitation target genes in LNCaP cells and LNCaP-AI cells. Histogram compares the genetic distribution in different biological processes between the cell lines. From the percentage of genes in the cells, it is clear that gene ontology (GO) terms in the LNCaP and LNCaP-AI cells are similar, although there a number of completely different GO terms within the biological process domain.

androgen-responsive genes in the LNCaP and LNCaP-AI cells, GO category enrichment analysis was applied using Fisher's exact test with an FDR cutoff adjusted to $\mathrm{P} \leq 0.01$. In the LNCaP cells, 1,797 genes were clustered into the biological process category, 1,996 genes were classified in the molecular function category and 2,061 genes were sorted into the cellular components domain. In the LNCaP-AI cells 1,231 genes were categorized into the biological process domain, 1,370 genes were sorted into the molecular function category and 1,414 genes were divided into the cellular components domain. In the biological process category, a number of different $\mathrm{GO}$ terms were identified between the $\mathrm{LNCaP}$ and LNCaP-AI cells, including the reproductive process, death, immune system process, multi-organism process, pigmentation and viral reproduction (Fig. 3). Table I lists the genes in the various GO terms. In the molecular function category, different GO terms associated with structural molecular activity, receptor regulator activity and antioxidant activity were identified between the LNCaP and LNCaP-AI cells. However, the GO terms in the cellular component domain were consistent between the LNCaP and LNCaP-AI cells.

Identification of AR enrichment consensus in the AR binding regions. To define whether the $\mathrm{AR}$ binding regions in $\mathrm{LNCaP}$ or LNCaP-AI cells have their own specificity and enriched binding motif, it was essential to rank all the peaks in $\mathrm{LNCaP}$ and LNCaP-AI cells according to enrichment, from highest to lowest. Subsequently, 100 peaks with a high level of statistical significance were identified in the two cell lines by analyzing the DNA-binding motifs using MEME software. In the LNCaP cells, six motifs were detected with a good statistical significance, while in the LNCaP-AI cells different sequences of six motifs were obtained. The Newick tree format is built based on the association between all 12 instances of motif, which resembles genealogy, as shown in Fig. 4. Using TOMTOM

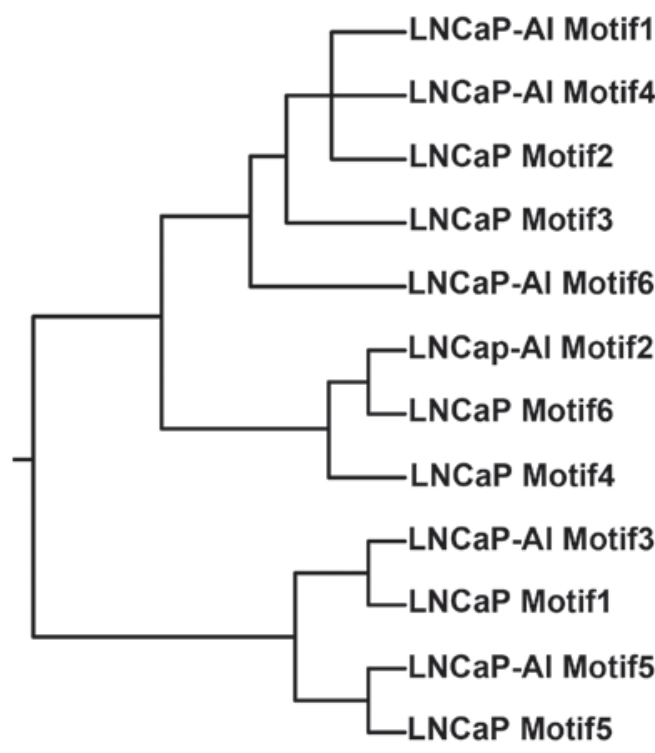

Figure 4. Motif tree. Each origin in the motif tree represents a motif, and each path in the motif tree represents a potential motif module.

software, it was easy to infer DNA-binding motifs from the JASPAR database, which shared a high level of homology with the 12 instances of motif obtained (Table II).

Categorization of AR binding regions identified by ChIP-seq based on their distribution to genes. The aim was to determine the location of the AR binding regions relative to the TSSs of the closet androgen-responsive genes. Subsequently, the frequency across the distance intervals prior to and subsequent to the TSS for every $2 \mathrm{~kb}$ were tabulated. Fig. 5 shows the peaks of the AR binding regions in the LNCaP and LNCaP-AI cells around the TSSs; a histogram of the $\mathrm{AR}$ binding regions residing within the downstream $50 \mathrm{~kb}$ 
Table II. Matrices were matched to each of the motifs in the JASPAR CORE database.

\begin{tabular}{llll}
\hline Name & \multicolumn{1}{c}{ Alignment } & Best match in JASPAR & Alignment (best match motif) \\
\hline LNCaP_Motif1 & GTGGATATTTGG & MA0130.1_ZNF354C & GTGGATATTTGG \\
LNCaP_Motif2 & AYRGARTKGAAY & MA0218.1_ct & RTTCMAYTCYRT \\
LNCaP_Motif3 & CAYTCYTTTTG & MA0277.1_AZF1 & CAAAARGARTG \\
LNCaP_Motif4 & AGTTTCTGAGAA & MA0432.1_YNR063W & TTCTCAGAAACT \\
LNCaP_Motif5 & GAGYNGWWTGGA & MA0371.1_ROX1 & GAGYNGWWTGGA \\
LNCaP_Motif6 & GCTTCTGTCTAG & MA0323.1_IXR1 & GCTTCTGTCTAG \\
LNCaP-AI_Motif1 & GASTTGAATGCA & MA0274.1_ARR1 & TGCATTCAASTC \\
LNCaP-AI_Motif2 & AGAATGCTTCTG & MA0417.1_YAP5 & CAGAAGCATTCT \\
LNCaP-AI_Motif3 & GTGGATATTTGG & MA0130.1_ZNF354C & CCAAATATCCAC \\
LNCaP-AI_Motif4 & ACAGAGTTGAAC & MA0218.1_ct & GTTCAACTCTGT \\
LNCaP-AI_Motif5 & GAGCAGTTTTGA & MA0299.1_GAL4 & GAGCAGTTTGA \\
LNCaP-AI_Motif6 & CGCTTTGAGGCC & MA0021.1_Dof3 & CGCTTTGAGGCC
\end{tabular}

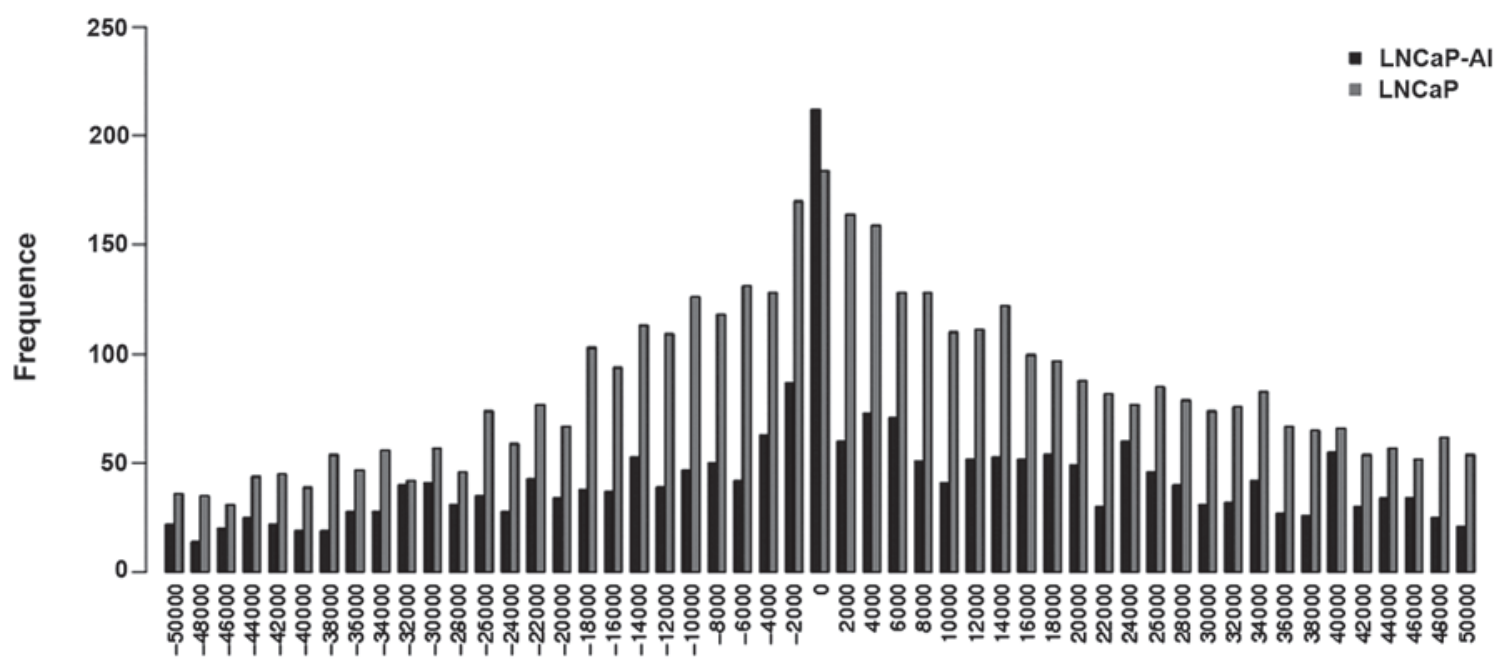

Figure 5. Histogram showing the androgen receptor binding sites around the annotated transcription start sites (TSSs) in LNCaP cells and LNCaP-AI cells. Frequencies of AR binding regions were calculated every $2 \mathrm{~kb}$ (y axis), and the relative distance to the TSS is shown on the $\mathrm{x}$ axis. Negative and positive values indicate $5^{\prime}$ or $3^{\prime}$ localization to the TSS, respectively.

or upstream $50 \mathrm{~kb}$ genomic regions relative to the annotated TSSs is shown. For the 4,143 AR binding regions in the LNCaP cells, $93 \%(3,871 / 4,143)$ resided within $50 \mathrm{~kb}$ of the TSSs. Similarly, for the 2,380 AR binding regions in the LNCaP-AI cells, $92 \%(2,185 / 2,380)$ resided within $50 \mathrm{~kb}$ of the TSSs. Among all the AR binding regions, $8.5 \%(354 / 4,143)$ were mapped to within $2 \mathrm{~kb}$ of the TSS in the LNCaP cells and $\sim 12.6 \%(299 / 2,380)$ were mapped to within $2 \mathrm{~kb}$ of the TSS in the LNCaP-AI cells.

\section{Discussion}

$\mathrm{AR}$ is a nuclear receptor that has been recognized as a major factor involved in prostate tumor genesis (20). A number of studies have shown that the majority of CRPC cases express AR and androgen-responsive genes $(21,22)$. The role of AR in prostate cancer progression is to promote the expression of specific target genes. The present study aimed to define AR binding sites in ligand-dependent and ligand-independent prostate cancer by performing ChIP-seq analysis in cell line models of ADPC and AIPC.

Genomic localization of the AR binding sites indicated that the majority of the androgen regulation was mediated by the binding of AR to the distal intragenic elements and intronic regions, as previously suggested $(11,12,23)$. A total of 2,796 and 1,854 actively transcribed target genes regulated by AR were identified in the LNCaP and LNCaP-AI cells, respectively. Comparisons between the cell lines demonstrated that there was a rare overlap of genes between the cell lines. The small overlap appeared to be a consequence of intrinsic differences, such as AR binding of other transcription factors (24). GO analysis of these genes revealed that there was significant overlap between $\mathrm{GO}$ terms in the LNCaP and LNCaP-AI cells. However, GO terms within the biological process domain that were only observed in LNCaP-AI cells included reproduction process, death, immune system process, multi-organism process, pigmentation and viral reproduction. The genes in the different GO terms included TNFAIP8, RTN4, APP and 
SYNE1 (Table I). TNFAIP8 is known to have oncogenic properties and is overexpressed in numerous cancer types $(25,26)$. Furthermore, TNFAIP8 expression is significantly associated with a higher risk of prostate cancer recurrence (27). A previous study demonstrated that APP expression levels correlated with the extent of disease and the prognosis in several forms of cancer (28). In addition, SYNE1 is a promising biomarker in colitis-associated colorectal cancer (29). As previously suggested, RTN4 contributes to the susceptibility of cervical squamous cell carcinoma (30).

In conclusion, these studies have provided new insights into the DNA sequences to which the AR can bind. Furthermore, AR cooperating transcription factors have been identified and thousands of potential AR-regulated genes have been mapped, providing insight into the biological processes regulated by the AR. In addition, the present study has added a number of candidate genes, including TNFAIP8, RTN4, APP and SYNE1 (Table I). However, further studies are required to clarify the function of the candidate genes, which may aid understanding into the molecular mechanisms underlying the transition from ADPC to AIPC. Subsequently, the candidate genes may aid the development of novel strategies for therapeutic intervention or serve as biomarkers of different cancer stages.

\section{Acknowledgements}

This study was supported by a grant from the National Natural Science Foundation of China (no. 81271917).

\section{References}

1. Suzuki H, Ueda T, Ichikawa T and Ito H: Androgen receptor involvement in the progression of prostate cancer. Endocr Relat Cancer 10: 209-216, 2003.

2. Debes JD and Tindall DJ: Mechanisms of androgen-refractory prostate cancer. N Engl J Med 351: 1488-1490, 2004.

3. Scher HI, Liebertz C, Kelly WK, et al: Bicalutamide for advanced prostate cancer: the natural versus treated history of disease. J Clin Oncol 15: 2928-2938, 1997.

4. Chen CD, Welsbie DS, Tran C, et al: Molecular determinants of resistance to antiandrogen therapy. Nat Med 10: 33-39, 2004.

5. Hu R, Dunn TA, Wei S, et al: Ligand-independent androgen receptor variants derived from splicing of cryptic exons signify hormone-refractory prostate cancer. Cancer Res 69: 16-22, 2009

6. Zeng R, Liu Z, Sun Y and Xu C: Differential expression and function of AR isoforms in prostate cancer. Oncol Rep 27: 492-498, 2012.

7. Carver BS, Chapinski C, Wongvipat J, et al: Reciprocal feedback regulation of PI3K and androgen receptor signaling in PTEN-deficient prostate cancer. Cancer Cell 19: 575-586, 2011.

8. Richter E, Srivastava S and Dobi A: Androgen receptor and prostate cancer. Prostate Cancer Prostatic Dis 10: 114-118, 2007.

9. Wang Q, Li W, Zhang Y, et al: Androgen receptor regulates a distinct transcription program in androgen-independent prostate cancer. Cell 138: 245-256, 2009.
10. Wang Q, Li W, Liu XS, et al: A hierarchical network of transcription factors governs androgen receptor-dependent prostate cancer growth. Mol Cell 27: 380-392, 2007.

11. Yu J, Yu J, Mani RS, et al: An integrated network of androgen receptor, polycomb, and TMPRSS2-ERG gene fusions in prostate cancer progression. Cancer Cell 17: 443-454, 2010.

12. Jia L, Berman BP, Jariwala U, et al: Genomic androgen receptor-occupied regions with different functions, defined by histone acetylation, coregulators and transcriptional capacity. PLoS One 3: e3645, 2008.

13. Xu G, Wu J, Zhou L, et al: Characterization of the small RNA transcriptomes of androgen dependent and independent prostate cancer cell line by deep sequencing. PLoS One 5: e15519, 2010.

14. Tewari AK, Yardimci GG, Shibata Y, et al: Chromatin accessibility reveals insights into androgen receptor activation and transcriptional specificity. Genome Biol 13: R88, 2012.

15. Zhang Y, Liu T, Meyer CA, et al: Model-based analysis of ChIP-Seq (MACS). Genome Biol 9: R137, 2008.

16. Bailey TL and Elkan C: Fitting a mixture model by expectation maximization to discover motifs in biopolymers. Proc Int Conf Intell Syst Mol Biol 2: 28-36, 1994.

17. Ma W, Noble WS and Bailey TL: Motif-based analysis of large nucleotide data sets using MEME-ChIP. Nat Protoc 9: 1428-1450, 2014.

18. Ashburner M, Ball CA, Blake JA, et al; The Gene Ontology Consortium: Gene ontology: tool for the unification of biology. Nat Genet 25: 25-29, 2000.

19. Tomlins SA, Mehra R, Rhodes DR, et al: Integrative molecular concept modeling of prostate cancer progression. Nat Genet 39: 41-51, 2007.

20. Vander Griend DJ, D'Antonio J, Gurel B, Antony L, Demarzo AM and Isaacs JT: Cell-autonomous intracellular androgen receptor signaling drives the growth of human prostate cancer initiating cells. Prostate 70: 90-99, 2010.

21. Kokontis JM, Hsu S, Chuu CP, Dang M, Fukuchi J, Hiipakka RA and Liao S: Role of androgen receptor in the progression of human prostate tumor cells to androgen independence and insensitivity. Prostate 65: 287-298, 2005.

22. Attard G, Richards J and de Bono JS: New strategies in metastatic prostate cancer: Targeting the androgen receptor signaling pathway. Clin Cancer Res 17: 1649-1657, 2011

23. Lupien M, Eeckhoute J, Meyer CA, et al: FoxA1 translates epigenetic signatures into enhancer-driven lineage-specific transcription. Cell 132: 958-970, 2008.

24. Zinzen RP, Girardot C, Gagneur J, Braun M and Furlong EE: Combinatorial binding predicts spatio-temporal cis-regulatory activity. Nature 462: 65-70, 2009.

25. Liu T, Gao H, Yang M, Zhao T, Liu Y and Lou G: Correlation of TNFAIP8 overexpression with the proliferation, metastasis, and disease-free survival in endometrial cancer. Tumour Biol 35: 5805-5814, 2014

26. Liu T, Xia B, Lu Y, Xu Y and Lou G: TNFAIP8 overexpression is associated with platinum resistance in epithelial ovarian cancers with optimal cytoreduction. Hum Pathol 45: 1251-1257, 2014.

27. Zhang C, Kallakury BV, Ross JS, et al: The significance of TNFAIP8 in prostate cancer response to radiation and docetaxel and disease recurrence. Int J Cancer 133: 31-42, 2013.

28. Chase D, McLauchlan G, Eckersall PD, Pratschke J, Parkin T and Pratschke K: Acute phase protein levels in dogs with mast cell tumours and sarcomas. Vet Rec 170: 648, 2012.

29. Papadia C, Louwagie J, Del Rio P, et al: FOXE1 and SYNE1 genes hypermethylation panel as promising biomarker in colitis-associated colorectal neoplasia. Inflamm Bowel Dis 20: 271-277, 2014.

30. Shi S, Zhou B, Wang Y, et al: Genetic variation in RTN4 3'-UTR and susceptibility to cervical squamous cell carcinoma. DNA Cell Biol 31: 1088-1094, 2012. 